\title{
Método Aprender a Crescer
uma nova perspectiva de crescimento para PMEs
}

Helio Aisenberg Ferenhof ${ }^{1}$ Bruna Devens Fraga ${ }^{2}$ Paulo Mauricio Selig ${ }^{3}$

\section{RESUMO}

As pequenas e médias empresas estão em uma constante busca de se manter no mercado e crescer. 0 método Aprender a Crescer ajuda nesta busca, este caracteriza-se por ser uma metodologia que procura integrar o planejamento estratégico da empresa por meio do desenvolvimento de projetos participativos elaborados juntamente com o gestor na forma de um processo de formação e aprendizagem. Com base nisso, este estudo objetivou responder a seguinte indagação: como melhorar as chances de uma PME em se manter no mercado e/ou crescer? Para isto, o presente estudo aplicou o método Aprender a Crescer (AaC) proposto por North (2012) com intuito de dar suporte a uma PME no segmento de consultoria. A aplicação do método iniciou a partir da escolha da organização em que seria aplicado o método. Após, foram seguidos os passos sugeridos pelo método: 1) Análise e identificação das lacunas e oportunidades de crescimento; 2) Proposição de projetos de aprendizagem, objetivando impulsionar as sugestões de melhoria; 3) Troca de experiências e melhores práticas advindas da experiência de aplicação do método; e por último, 4) Formação coachs do crescimento dentro da organização analisada. A microempresa escolhida atua no setor de prestação de serviços desenvolvendo projetos em diversas áreas e setores de gestão e tecnologia da informação. Dentre os resultados obtidos com a aplicação do método Aprender a Crescer constatou-se que a empresa encontrase em processo de maturação e questões fundamentais estratégicas, como estabelecimento de fontes de recursos a longo e alianças institucionais que permitam maior estabilidade, ainda necessitam ser alinhadas para obter um crescimento sustentável.

Palavras-Chave: PME. Método Aprender a Crescer. AaC. Crescimento. Inovação. 


\section{INTRODUÇÃO}

Com base nos cálculos da Organização para a Cooperação e Desenvolvimento Econômico (OCDE), Veronesi (2013) afirma que as PMEs representam 99\% das empresas registradas no País e detém 52\% da força de trabalho. Nos últimos anos, a taxa de sobrevivência das PMEs melhorou, passando de 50\%, no começo da década, aos 73\% atuais. 0 índice supera o de países como Itália (68\%) e Espanha (69\%).

Há um certo senso comum a caracterizar o perfil das pequenas e médias empresas (PMEs) do País. Estes são traços típicos que as qualificam, por exemplo, como organizações predominantemente familiares, de sociedade limitada e sediadas nas proximidades dos grandes centros econômicos (DELOITTE, 2008).

Ao experimentarem um ciclo evolutivo de seus negócios nos últimos anos, as PMEs se tornaram demandantes, de forma mais intensa, de serviços do sistema financeiro para darem continuidade ao seu crescimento (DELOITTE, 2008). Contudo, nem sempre essa estratégia garante uma estabilidade para a empresa, ou ainda fornece um meio de garantir um desenvolvimento adequado a longo prazo. Desta forma, outras PMEs procuram recursos em editais de pesquisa em instituições de fomento ou ainda junto a universidades e incubadoras de pesquisa e desenvolvimento.

Um dos principais atributos de empresas que se mostram sempre preparadas para eventuais transformações na dinâmica dos negócios é a busca constante pela eficiência (DELOITTE, 2009). Por isso, a descoberta e o desenvolvimento de mecanismos geradores de eficiência são desafios que marcam a gestão das PMEs que mais crescem no atual contexto.

Sendo assim, como melhorar as chances de uma PME em se manter no mercado e/ou crescer? Com base nesta indagação, o presente estudo aplicou o método Aprender a Crescer (AaC) proposto por North (2012) com intuito de dar suporte a uma PME no segmento de consultoria. De modo a ensinar a esta a identificar seu estado atual, suas oportunidades de melhorias e assim, traçar ações estratégicas alinhadas ao seu mercado. Para atingir este objetivo o presente estudo apresenta-se em cinco seções.

A seção dois se destina a descrever o método Aprender a Crescer, por sua vez, a seção três apresenta a metodologia de pesquisa adotada neste estudo. A seção quatro narra a aplicação do AaC. E por fim, são expostas as considerações finais e proposições futuras na seção cinco.

\subsection{0 método Aprender a Crescer $(\mathrm{AaC})$}

O método Aprender a Crescer desenvolvido por North (2012) objetiva a integração do planejamento estratégico da empresa por meio da execução de um projeto estratégico com um processo de formação e aprendizagem. 0 intuito desta ação é capacitar as empresas desenvolver habilidades e competências a fim de reconfigurá-las diante que qualquer circunstância e momento, criando valor ao cliente por intermédio da inovação. 0 método é desenhado em quatro passos: 1) Identificação de oportunidades e problemas de crescimento; 2) Criação de Projetos de Aprendizagem, que tem o intuito de desenvolver as competências do capital intelectual da empresa bem como materializar ações impulsionadoras. 3) Compartilhamento das experiências e melhores práticas obtidas pela aplicação do método; 4) Formação de técnicos (coachs) do crescimento dentro das empresa, por meio das experiências adquiridas na execução dos Projetos de Aprendizagem.

\subsection{Como se aplica?}

A aplicação do método é melhor entendida pela figura 1- Roda do Crescimento, que apresenta as alavancas do crescimento, no total de oito, e os quatro desafios a serem alcançados. Estão associadas a cada uma dos quatro desafios, duas alavancas. 


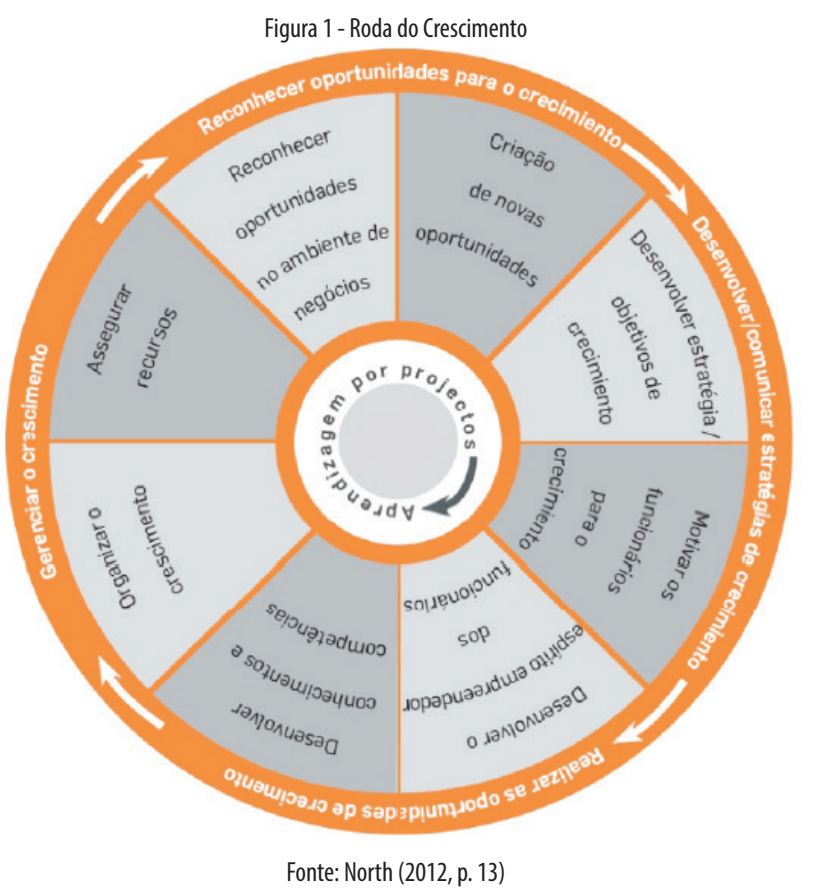

De acordo com Hardwig, Bergstermann e North (2011), para se aplicar o método é preciso seguir um ciclo de sete passos: o primeiro passo se consiste em analisar a capacidade de crescimento e reconhece as oportunidades de melhoria na empresa por meio de diagnóstico. 0 segundo passo é definir um projeto estratégico de crescimento, com base no diagnóstico previamente elaborado. Por sua vez, o terceiro passo é definir a matriz de responsabilidade de quem executará as tarefas relacionadas ao projeto a um grupo de funcionários. 0 quarto passo é identificar e capturar as oportunidades de crescimento. 0 quinto passo se destina a desenvolver as competências da equipe e de cada um dos seus integrantes. Por meio do entendimento das oportunidades capturadas previamente. No sexto passo se avalia os resultados obtidos na execução do projeto, gerando lições aprendidas. Finalmente no sétimo passo todos participam em reuniões de intercâmbio de experiências e melhores práticas aplicadas, constituindo uma forma de aprendizagem organizacional e interorganizacional, caso seja aplicada a mais de uma empresa em conjunto. Os passos podem ser melhor visualizados na Figura 2.

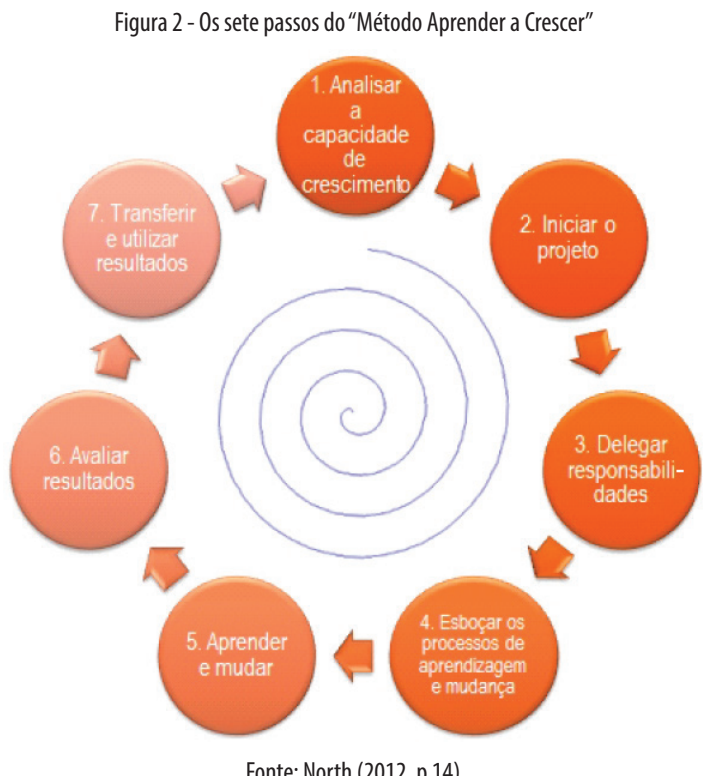




\section{METODOLOGIA DE PESQUISA}

O presente estudo de natureza empírica realizou-se por meio da pesquisa-ação. Esta caracteriza-se por ser uma forma de investigação autorreflexiva realizada pelos participantes em situações sociais com intuito de: melhorar a racionalidade e a justiça de suas próprias práticas; melhorar a sua compreensão dessas práticas e; melhorar as situações em que as práticas são realizada (CARR; KEMMIS, 1986).

Assim, a pesquisa-ação tem bases em um sistema de comunicação dialógica entre os envolvidos e pesquisadores onde todos são sujeitos da pesquisa, participando ativamente para um determinado fim, para a produção de um novo tipo de conhecimento dentro de um determinado contexto (GIL, 2002). 0 que está diretamente alinhado a aplicação do método Aprender a Crescer.

\section{APLICAÇÃO DO AAC}

Desde o início de sua atuação no Mercado, em 2005, a empresa estudada tem procurado estabelecer fortes laços com seus Clientes, desenvolvendo um relacionamento baseado em parceria e confiança, respaldado pela qualidade dos serviços prestados.

Ela tem atuado intensamente na área de prestação de serviços, desenvolvendo em conjunto com o Cliente soluções abrangentes para a otimização e crescimento do negócio. São projetos envolvendo aspectos diversos da prática de Gestão do Conhecimento, Gerenciamento de Projetos e Consultoria em Tecnologia de Informação, atendendo a empresas de pequeno, médio e grande porte pertencentes a variados setores da economia.

\section{1 Descrição empresa}

A empresa estudada surgiu da experiência de seu sócio-diretor que atua no mercado de TI e Gerenciamento de Projetos desde 1992. Passando por diversas empresas nacionais e multinacionais de renome, tendo passado por diversos cargos e funções desde programador, instrutor de cursos, analista de sistemas, gerente de projetos, gerente de TI.

A visão da empresa é garantir a entrega de serviços de excelência, dentro dos prazos, custos e qualidade acordados, excedendo os níveis de produtividade. Visando a segurança da informação e otimização dos processos envolvidos.

O alto padrão de atendimento vem dos constantes investimentos em Certificações e metodologias.

A empresa conta em seu quadro de prestadores de serviços, com profissionais com ampla experiência de mercado e com respaldo de suas devidas Certificações nas mais diversas áreas.

\subsection{Roda da metodologia}

A partir da Figura 1 - Roda do Crescimento foi feito o diagnóstico com o diretor-presidente por meio de entrevistas que auxiliaram a compor o quadro 1.

A cada desafio do crescimento, são determinadas duas alavancas. A cada alavanca, são determinadas duas ações.

Cada ação corresponde a uma pergunta do diagnóstico, que deve ser aplicado com o gestor. Essas ações possuem dois fatores que devem ser preenchidos pelas entrevistas: as cores (vermelho, amarelo e verde) e os valores $(0,1,2,3)$. As cores dizem respeito ao quanto à organização executa cada ação: Verde - Fazemos isso bem; Amarelo - Fazemos isso mais ou menos; Vermelho - Nós não fazemos isso bem. Já a numeração corresponde ao grau de relevância para a competitividade, que o gestor considera como de maior valor para sua organização. Por exemplo, 0 (zero) corresponde a uma ação que "não é relevante", 1 equivale a "tem pouca relevância", 2 "é relevante", e o 3 quando "é altamente relevante".

Logo, a cada ação são preenchidos esses requisitos que no final irão compor o diagnóstico da Roda do Crescimento. Feito o levantamento que revela o grau de execução da organização e a relevância para cada ação, é feito uma média para descobrir a localização de cada ação no plano cartesiano de diagnóstico, como mostra o Quadro 1. 
Quadro 1 - Roda do Conhecimento

\begin{tabular}{|c|c|c|c|c|c|c|c|}
\hline \multirow{4}{*}{$\begin{array}{l}\text { Reconhecer } \\
\text { oportunidades } \\
\text { para } 0 \\
\text { crescimento }\end{array}$} & \multirow{2}{*}{$\begin{array}{l}\text { Reconhecer } \\
\text { oportunidades no } \\
\text { ambiente de negócios }\end{array}$} & $\begin{array}{l}\text { 1. Nossa empresa reconhece sistematicamente } \\
\text { oportunidades e tendências nos mercados de referência } \\
\text { e capta sistematicamente os conhecimentos externos de } \\
\text { fornecedores, parceiros, clientes. }\end{array}$ & & $X$ & & 3 & \multirow[t]{2}{*}{3} \\
\hline & & $\begin{array}{l}\text { 2. Nossa empresa é capaz de captar de forma sistemática as } \\
\text { ideias dos funcionários. }\end{array}$ & $X$ & & & 3 & \\
\hline & \multirow{2}{*}{$\begin{array}{l}\text { Criação de novas } \\
\text { oportunidades }\end{array}$} & $\begin{array}{l}\text { 3. Nossa empresa orienta suas atividades voltadas ao cliente } \\
\text { e cria novas oportunidades com base em necessidades } \\
\text { (futura) dos clientes. }\end{array}$ & $x$ & & & 3 & \multirow[b]{2}{*}{2,5} \\
\hline & & $\begin{array}{l}\text { 4. Através de parcerias e redes com parceiros externos } \\
\text { (instituições de pesquisa, universidades, associações, etc.) } \\
\text { sistematicamente criamos oportunidades para inovaçãa e } \\
\text { crescimento. }\end{array}$ & & & $x$ & 2 & \\
\hline \multirow{4}{*}{$\begin{array}{l}\text { Desenvolver/ } \\
\text { comunicar } \\
\text { estratégias de } \\
\text { crescimento }\end{array}$} & \multirow{2}{*}{$\begin{array}{l}\text { Desenvolver } \\
\text { estratégia/ objetivos } \\
\text { de crescimento }\end{array}$} & $\begin{array}{l}\text { 5. Temos uma estratégia e objetivos de crescimento } \\
\text { convincentes. Nós somos capazes de atualizar a estratégia } \\
\text { e os objetivos para responder rapidamente a novas } \\
\text { tendências. }\end{array}$ & & & $x$ & 2 & \multirow{4}{*}{2} \\
\hline & & $\begin{array}{l}\text { 6. Estamos aptos a desenvolver novos modelos de negócios } \\
\text { que criam valor a longo prazo. }\end{array}$ & & & $X$ & 2 & \\
\hline & \multirow[t]{2}{*}{$\begin{array}{l}\text { Motiva os } \\
\text { funcionários para } 0 \\
\text { crescimento }\end{array}$} & $\begin{array}{l}\text { 7. A estratégia corporativa não existe apenas na mente } \\
\text { do empresário, mas é comunicada intensamente e os } \\
\text { funcionários estão envolvidos em seu desenvolvimento } \\
\text { entendendo como podem contribuir para o sucesso do } \\
\text { negócio. }\end{array}$ & $x$ & & & 3 & \\
\hline & & $\begin{array}{l}\text { 8. Nossa empresa sabe como entusiasmar seus empregados } \\
\text { em relação aos objetivos da empresa. }\end{array}$ & & $X$ & & 1 & \\
\hline \multirow{4}{*}{$\begin{array}{l}\text { Realizar as } \\
\text { oportunidades de } \\
\text { crescimento }\end{array}$} & \multirow{2}{*}{$\begin{array}{l}\text { Desenvolver o espírito } \\
\text { empreendedor dos } \\
\text { funcionários }\end{array}$} & $\begin{array}{l}\text { 9. Nossos funcionários pensam e agem com o } \\
\text { espírito empresarial e assumem um elevado grau de } \\
\text { responsabilidade. }\end{array}$ & & $X$ & & 1 & \multirow[t]{2}{*}{1} \\
\hline & & $\begin{array}{l}\text { 10. Nossos executivos sabem delegar e criar espaços de } \\
\text { desempenho ("empowerment") para os funcionários. }\end{array}$ & $x$ & & & 1 & \\
\hline & \multirow{2}{*}{$\begin{array}{l}\text { Desenvolver } \\
\text { conhecimento e } \\
\text { competências }\end{array}$} & $\begin{array}{l}\text { 11. Sistematicamente desenvolvemos as competências dos } \\
\text { nossos funcionários para garantir crescimento. }\end{array}$ & & $x$ & & 2 & \multirow[b]{2}{*}{2,5} \\
\hline & & $\begin{array}{l}\text { 12. Compartilhamos ativamente os conhecimentos entre } \\
\text { os departamentos, funçôes e através de várias hierarquias } \\
\text { na empresa. }\end{array}$ & $X$ & & & 3 & \\
\hline \multirow{4}{*}{$\begin{array}{l}\text { Gerenciar } 0 \\
\text { crescimento }\end{array}$} & \multirow{2}{*}{$\begin{array}{l}\text { Organizar } 0 \\
\text { crescimento }\end{array}$} & $\begin{array}{l}\text { 13. Somos capazes de adaptar os nossos processos e a } \\
\text { organização (empresa) para os desafios do crescimento. }\end{array}$ & & $X$ & & 2 & \multirow{2}{*}{2} \\
\hline & & $\begin{array}{l}\text { 14. Executivos e funcionários sabem conviver com } 0 \\
\text { crescimento. }\end{array}$ & & & $X$ & 2 & \\
\hline & \multirow{2}{*}{ Assegurar recursos } & $\begin{array}{l}\text { 15. Asseguramos a disponibilidade de funcionários } \\
\text { necessários para } 0 \text { crescimento. }\end{array}$ & & & $X$ & 3 & \multirow{2}{*}{3} \\
\hline & & $\begin{array}{l}\text { 16. Asseguramos o financiamento do crescimento de forma } \\
\text { proativa. }\end{array}$ & & $X$ & & 3 & \\
\hline
\end{tabular}

Fonte: autores, com base em North (2012)

\section{3 Quadro diagnóstico}

O quadro diagnóstico proposto na Metodologia Aprender a Crescer é composto por um plano cartesiano que pontua duas dimensões: Habilidade da Empresa e Relevância Estratégica. Estas revelam os pontos chave que devem ser explorados para ajudar a organização no seu crescimento. É quase um "espelho" que retrata a atual situação em que se encontra e pontua as suposições que merecem atenção ou mesmo transformadas. 


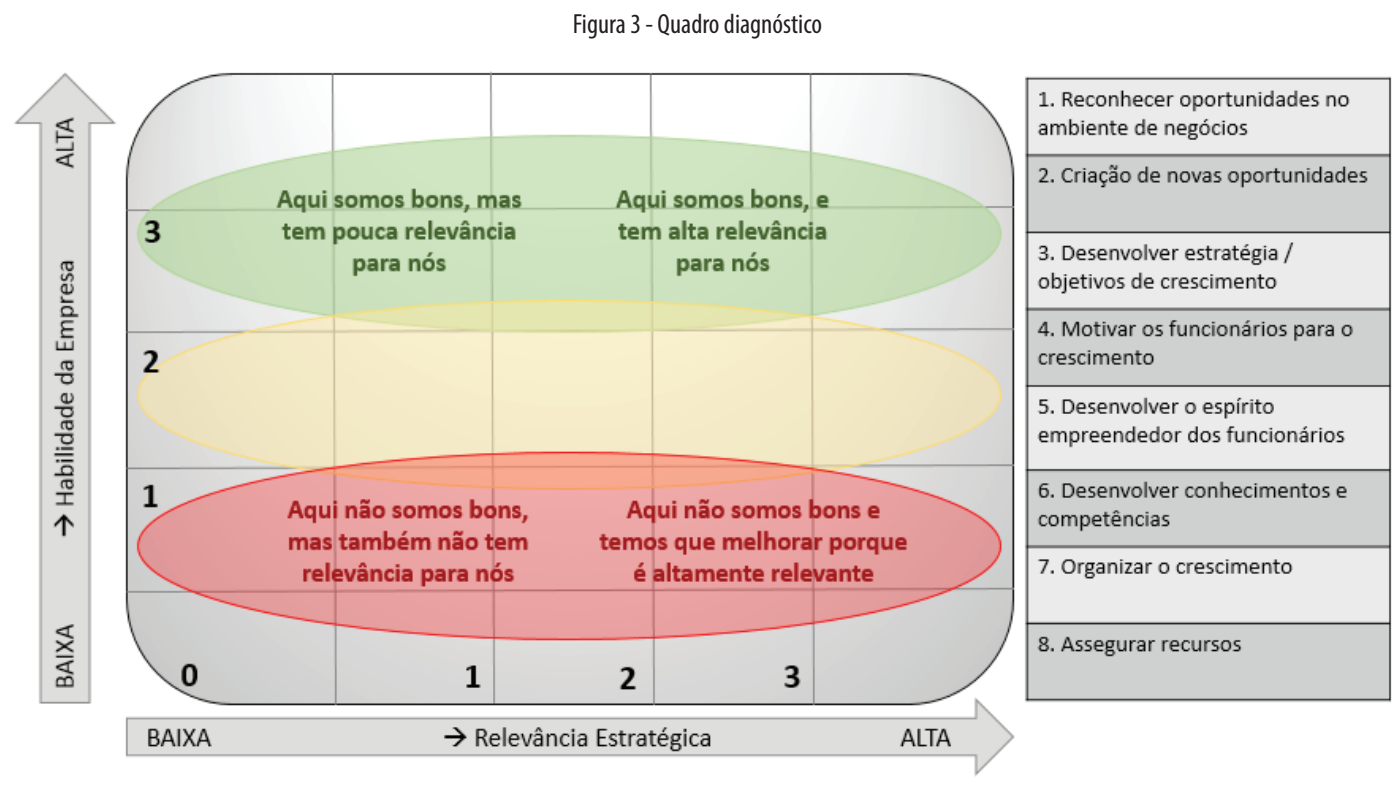

Fonte: autores, com base em North (2012)

Com base no Plano Cartesiano (Figura 3), foi desenhado os pontos críticos apontados por meio do diagnóstico da Roda do Crescimento, como mostra a figura 4 - Aplicação do quadro diagnóstico.

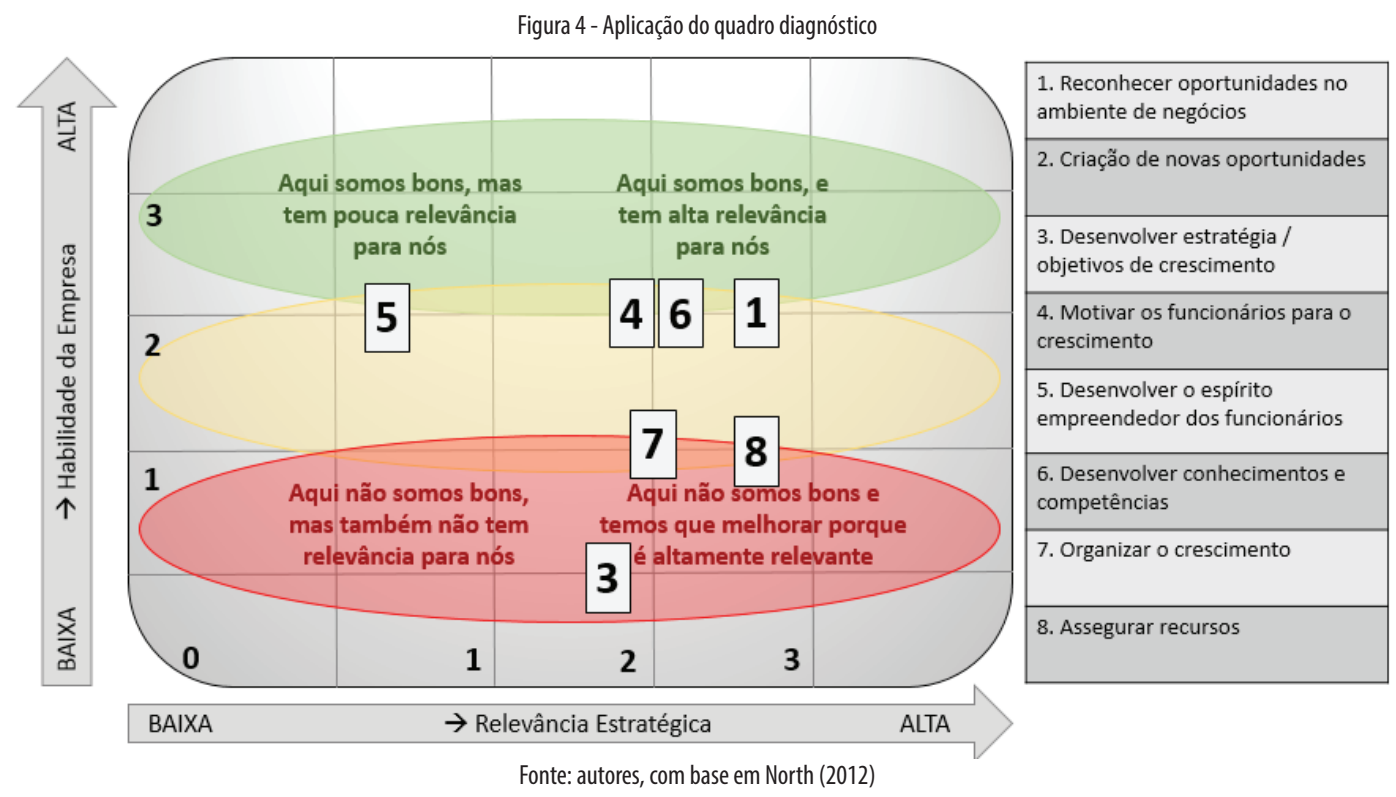

\subsection{Situação atual $x$ ideias de melhoria}

Diante dos pontos relevantes apontados na figura 4, que dizem respeito aos aspectos que devem ser melhorados devido à relevância que representam para a organização, foram levantados alguns dos principais questionamentos feitos ao gestor e foram apontadas sugestões de melhoria para o projeto final.

- Através de parcerias e de redes com parceiros externos (instituições de pesquisa, universidades, associações, etc.) sistematicamente criamos oportunidades para inovação e crescimento. 
Quadro 2 - Diagnóstico de Situação Atual e Proposta de Melhoria

\begin{tabular}{|c|c|}
\hline Situação Atual & Ideias de Melhoria \\
\hline $\begin{array}{c}\text { Problema crítico para o desenvolvimento da organização. } \\
\text { Houveram tentativas com diversos possíveis parceiros. } \\
\text { Principais: alianças com consultoria e treinamento. }\end{array}$ & $\begin{array}{c}\text { Aumentar números de parceiros, como instituições } \\
\text { de pesquisa que são um campo de aplicação e } \\
\text { desenvolvimento de métodos e técnicas. } \\
\text { Cadastrar junto à base de empresas do SEBRAE, e } \\
\text { assim realizar o devido monitoramento de editais. }\end{array}$ \\
\hline
\end{tabular}

Fonte: autores, com base em North (2012)

- $\quad$ Estamos aptos a desenvolver novos modelos de negócios que criam valor a longo prazo.

Quadro 3 - Diagnóstico de Situação Atual e Proposta de Melhoria

\begin{tabular}{|c|c|}
\hline Situação Atual & Ideias de Melhoria \\
\hline $\begin{array}{c}\text { A empresa está em processo de maturidade. Por ser micro, no } \\
\text { momento não é o foco, pois depende do fluxo de caixa. }\end{array}$ & $\begin{array}{c}\text { Participar do PRIME (Primeira Empresa Inovadora) } \\
\text { Submeter projeto ao FINEP (Financiadora de Estudos } \\
\text { e Projetos) e outros órgãos de fomento. }\end{array}$ \\
\hline
\end{tabular}

Fonte: autores, com base em North (2012)

- A estratégia corporativa não existe apenas na mente do empresário, mas é comunicada intensamente e os funcionários estão envolvidos em seu desenvolvimento entendendo como podem contribuir para o sucesso do negócio.

Quadro 4 - Diagnóstico de Situação Atual e Proposta de Melhoria

\begin{tabular}{|c|c|}
\hline Situação Atual & Ideias de Melhoria \\
\hline $\begin{array}{c}\text { A empresa depende apenas do proprietário. } \\
\text { Funcionários terceirizados, impede crescimento. }\end{array}$ & $\begin{array}{c}\text { Fluxo de caixa contínuo para assumir contratação } \\
\text { efetiva de funcionários que se engajem nas diretrizes } \\
\text { da empresa. }\end{array}$ \\
\hline
\end{tabular}

Fonte: autores, com base em North (2012)

Diante dos apontamentos sugeridos segundo a ferramenta da Metodologia Aprender a Crescer, como resultado desse processo foi proposto um projeto que vise auxiliar a organização no seu desenvolvimento e crescimento a longo prazo.

\subsection{Criação de projeto de aprendizagem}

Conforme apontam os objetivos da metodologia os seguintes passos devem ser seguidos:

a) Realizar diagnóstico do crescimento e definir objetivos estratégicos de crescimento e determinar a alavanca de crescimento.

b) Definir o projeto de crescimento e articular as expectativas para o desenvolvimento da flexibilidade ao ambiente e ao mercado e o desenvolvimento de competências da organização.

c) Estimar a necessidade de aprendizagem dependendo das expectativas declaradas.

Segundo as proposições de melhoria diagnosticadas e sugeridas pelo próprio diretor-presidente e também pelo coordenador de aplicação da Metodologia Aprender a Crescer, foi elaborado um projeto seguindo as diretrizes da organização. A proposta do projeto baseia-se no desenvolvimento de produto (bem/serviço) com recursos próprios (parcerias/ faturamento) ou via fontes de fomento (FINEP/ FAPESC/ SEBRAE).

De acordo com as necessidades levantadas, um dos problemas críticos está na razão da centralização do planejamento e execução dos trabalhos no diretor-presidente que também é o único efetivo da empresa. É preciso um incentivo na busca de novos projetos de longa duração que promovam a integração de novos membros no funcionamento e execução dos projetos que surgem.

Essa captação é essencial para movimentar o fluxo de caixa da organização, que no momento encontrase estagnado. 0 aprimoramento das técnicas e métodos elaborados pela organização é crucial para atrair novos clientes e ganhar visibilidade no mercado. 


\subsection{Compartilhamento das experiências e melhores práticas}

Por meio da aplicação do método se identificou os principais gaps e as formas de como saná-los. As experiências obtidas tanto no processo de identificação quanto no processo de solução foram compartilhadas em reuniões específicas de lições aprendidas. Estas, podem ser definidas como as experiências chave que contêm certa relevância de negócios para projetos futuros (SCHINDLER; EPPLER, 2003; FERENHOF; FORCELLINI; VARVAKIS, 2011).

Ressalta-se que sob o olhar da gestão do conhecimento, toda e qualquer experiência é conhecimento e este, deve ser explicitado, compartilhado e disseminado para agregar valor as pessoas e organizações (FERENHOF; FORCELLINI; VARVAKIS, 2011).

\subsection{Formação de técnicos (coachs) do crescimento}

O método $\mathrm{AaC}$ proporcionou a indicação e formação de responsáveis por ensinar os procedimentos internos de potencial crescimento da empresa estudada. Por meio das experiências adquiridas na execução dos Projetos de Aprendizagem foi possível a identificação do profissional com melhor perfil indicado para se tornar coach do crescimento. Não eximindo a responsabilidade dos demais membros da empresa em participar do processo ensino-aprendizagem. Representando ora o papel de aprendiz, ora de treinador.

Esta etapa ainda encontra-se em fase de maturação, em decorrência do projeto ainda estar em fase de desenvolvimento. Contudo, o resultado obtido da troca de experiências e melhores práticas entre os membros da organização vem contribuindo para a prospecção de projetos futuros alinhados à adequação das lacunas identificadas na aplicação do método AaC.

\section{CONSIDERAÇÕES FINAIS}

O método AaC colabora com a identificação de gaps e oportunidades de crescimento para as PMEs, por meio de ferramentas e quadros que suportam uma participação efetiva do gestor na fase de diagnóstico da sua organização. As ferramentas que o método aponta, facilitam a comunicação de forma visual de como a organização está se desenvolvendo e em quais fatores críticos que a empresa deve trabalhar para obter um crescimento efetivo e sustentável.

Com base nestes aspectos, o gestor passa a ter um olhar holístico da empresa, não apenas para os recursos financeiros, aprimorando os aspectos humanos e relacionais, no que dizem respeito à inovação e competitividade. Por meio da Roda do Crescimento, o gestor passa a identificar o mapa de relacionamentos da empresa, funcionários e parceiros como parte integrante do crescimento organizacional. Desta forma, as PMEs passam a incorporar em suas concepções gerenciais a accountability necessária para promover o seu desenvolvimento a longo prazo.

Além de buscar competitividade, as PMEs precisam passar a entender suas necessidades presentes e futuras, prospectando um melhor planejamento estratégico de modo a traçar ações para a manutenção e crescimento da empresa.

O que se pode considerar com a aplicação do método na empresa estudada, que o método AaC ajudou a direção a tomar ciência da situação atual, em que a empresa encontra-se em processo de maturação e crescimento, identificando oportunidades de melhorias tais como: 1) a empresa deve estabelecer parcerias (tanto para obter recursos, quanto para desenvolvimento conjunto de atividades); 2) a necessidade de uma fonte financeira a longo prazo; 3) busca de projetos com maior duração, garantindo assim recursos financeiros mais longos; 4) desenvolver um produto à ser comercializado para garantir de forma recorrente de recursos financeiros.

Como proposição de ações futuras vem o acompanhamento da implantação das ações recomendadas pelo método, bem como a execução da roda mais de uma vez após as implantações. Outra recomendação futura é aplicar o método em mais de uma empresa seja de setores iguais ou diferentes e acompanhar os resultados de crescimento destas. 


\title{
THE METHOD “LEARN TO GROWTH": A NEW PERSPECTIVE FOR SMES GROWTH
}

\begin{abstract}
Small and medium enterprises are in a constant search to maintain and grow. The Learn to Grow method helps in this search. It's characterized by being a methodology that integrates the company's strategic planning with the development of a project that align the training and learning with the participation of the managers, sponsor and staff. Based on this context, this study aimed to answer the following question. How to improve the chances of SMEs in maintaining the market and / or grow? To answer this issue, this study applied the method Learning to Grow proposed by North (2012) aiming to support a SME serviceconsulting firm. This work applied the method following the steps suggested by the method: 1) Analysis and identification of gaps and opportunities for growth; 2) Proposition learning projects, with the aim of boosting suggestions for improvement; 3) Exchange of experiences and best practices arising from experience application of the method, and finally, 4) Training coach's to growth within the organization analyzed. The SME chosen operates in the provision of services by developing projects in several areas and sectors of management and information technology. Among the results obtained from the application of the method "Learn to Growth" it was found that the company is in the process of maturation. By that, fundamental strategic issues such as the establishment of funding sources and long institutional alliances is necessary to allow greater stability to achieve sustainable growth.
\end{abstract}

Keywords: SME. Learn to Grow method. Growth. Innovation.

\section{REFERÊNCIAS}

CARR, W.; KEMMIS, S. Becoming Critical: Education, Knowledge and Action Research. London: Falmer Press, 1986.

DELOITTE, T. T. As pequenas e médias empresas que mais crescem no Brasil: uma pesquisa sobre as rotas e estratégias que levam à expansão dos negócios. [2008]. Disponível em: <http://www.deloitte.com/ assets/Dcom- Brazil/Local\%20Assets/Documents/PesqExame2008.pdf>. Acesso em: 26 jul. 2013.

DELOITTE, T. T. Eficiência nos novos tempos da economia. Um estudo sobre as pequenas e médias empresas que mais crescem no Brasil. [2009]. Disponível em: <http://www.deloitte.com/assets/DcomBrazil/Local\%20Assets/Documents/Relatorio\%20PME\%20Deloitte\%202009.pdf>. Acesso em: 26 jul. 2013.

FERENHOF, H. A.; FORCELLINI, F. A.; VARVAKIS, G. Lições aprendidas: agregando valor ao gerenciamento de projetos. In: Conferência Internacional de Gerenciamento de Projetos, 2011, Florianópolis. Anais... Florianópolis: PMI/SC, 2011.

GIL, A. C. Como elaborar projetos de pesquisa. 4. ed. São Paulo: Atlas, 2002.

HARDWIG, T.; BERGSTERMANN, M.; NORTH, K. Wachstum lernen. Wiesbaden: Gabler, 2011.

NORTH, K. Aprender a Crescer: Apresentação em formato de mídia. Wiesbaden: Wiesbaden Business School, 2012.

SCHINDLER M.; EPPLER M. J. Harvesting project knowledge: a review of project learning methods and success factors International. International Journal of Project Management, Philadelphia, v. 21, n. 3, p. 219-228, Apr. 2003.

VERONESI, L. B. Santander irá investir R 20 bilhões em PMEs brasileiras [2013]. Disponível em: <http://www.infomoney.com.br/negocios/emprestimos/noticia/2841801/santander-ira-investirbilhoes-pmes-brasileiras>. Acesso em: 26 jul. 2013. 